\title{
The German version of the Body Image Scale: Validation among a sample of cancer survivors
}

\author{
Tim J. Hartung, $\mathrm{MD}^{1,2}$, Michael Friedrich, $\mathrm{PhD}^{1}$, Peter Esser, $\mathrm{PhD}^{1}$, \\ Anja Mehnert-Theuerkauf, $\mathrm{PhD}^{1}$, Antje Lehmann-Laue, $\mathrm{PhD}^{1}$, Anne Letsch, $\mathrm{MD}^{3,4}$, \\ Matthias Rose, $\mathrm{MD}^{5,6}$, Uta Fendel, $\mathrm{MD}^{5}$
}

\footnotetext{
${ }^{1}$ Department of Medical Psychology and Medical Sociology, University Medical Center Leipzig, Leipzig, Germany

${ }^{2}$ Department of Neurology, Charité - Universitätsmedizin Berlin, Corporate Member of Freie Universität Berlin and Humboldt-Universität zu Berlin, Berlin, Germany

${ }^{3}$ Department of Hematology and Oncology, Campus Benjamin Franklin, Charité-Universitätsmedizin Berlin, Corporate Member of Freie Universität Berlin, Humboldt-Universität zu Berlin, and Berlin Institute of Health, Berlin, Germany

${ }^{4}$ Department of Medicine II, Hematology and Oncology, University Hospital Schleswig-Holstein, Kiel, Germany

${ }^{5}$ Department of Psychosomatic Medicine, Charité - Universitätsmedizin Berlin, Corporate Member of Freie Universität Berlin and Humboldt-Universität zu Berlin, Berlin, Germany

${ }^{6}$ Department of Quantitative Health Sciences, Medical School, University of Massachusetts, Amherst, Massachusetts
}

A peer reviewed version of this article has been published as:

Hartung TJ, Friedrich M, Esser P, Mehnert-Theuerkauf A, Lehmann-Laue A, Letsch A, Rose M, Fendel U. The German version of the body image scale: Validation and item response analysis in cancer patients. J Psychosom Res. 2022 Dec 23;165:111129.

doi: 10.1016/j.jpsychores.2022.111129.

Corresponding author: Tim J. Hartung, tim.hartung@medizin.uni-leipzig.de

Supplemental files at: https://osf.io/8uecm/

Conflicts of Interest: The authors have declared no conflict of interest.

Source of Funding: No external funding was used for this study.

Acknowledgements:

We thank Hui Zhang, Mikaela Lui and Amber McPherson for their translations and David Kissane for comparing the back translations with the original version. Many thanks to Larissa Rudolph for her help with data entry. We are also indebted to Penelope Hopwood for the fruitful discussions regarding this study and her very valuable and helpful comments and suggestions. 
Key words (MeSH):

Body Image

Neoplasms

Psychometrics

Translations

Factor Analysis

Statistical

\section{Acronyms:}

AIC, Akaike's Information Criterion

AVE, average variance extracted

BAS, Body Appreciation Scale

BID, body image disturbance

BIS, Body Image Scale

CFA, confirmatory factor analysis

CFI, comparative fit index

CI, confidence interval

$\mathrm{CR}$, composite reliability

DT, Distress Thermometer

GAD-2, Generalized Anxiety Scale 2-item version

ICD, International Classification of Diseases

IRT, item response theory

$\mathrm{M}$, mean

PHQ-9, Patient Health Questionnaire 9-item depression screener

RMSEA, Root Mean Square Error of Approximation

SD, standard deviation

SMC, squared multiple correlations

SMC, squared multiple correlations

SRMR; standardized root mean square residual

TLI, Tucker-Lewis-Index 


\section{ABSTRACT}

Objective: To translate the cancer-specific Body Image Scale (BIS) into German and to validate the translated version by assessing its psychometric properties.

Methods: The state-of-the-art translation of the BIS was tested in (i) a psychosocial cancer counselling center in Leipzig and (ii) oncological wards in Berlin, Germany. Composite reliability and factor structure were investigated using confirmatory factor analysis. Item response theory (IRT)-based analyses were performed. Convergent validity was assessed via correlation with another body image questionnaire (BAS) as well as psychological symptom burden (PHQ-9, GAD2 and DT). Psychosocial and clinical group differences were assessed using t-tests.

Results: 677 patients participated (response rate 78\%). Composite reliability was 0.92 and the average variance extracted was above 50\%. All items had a discriminating power above the established threshold of $b=0.5$ and showed optimal discriminatory power at relatively high severity levels of body image disturbance (BID) $(b=0.89-2.06)$. Test information was highest in patients with moderate BID $(\theta=1.36)$. The BIS was negatively correlated with the BAS $(r=-0.62, p<$ 0.001) and positively with psychological symptom burden (e.g. PHQ-9: $r=0.49, \mathrm{p}<0.001$ ). Patients who were younger, female, had undergone chemotherapy and those who were distressed by fatigue, their appearance or sexual problems had significantly higher BID.

Conclusions: The German version of the BIS is a valid tool to assess BID in patients with cancer. Normative data are needed to better interpret individual scores within the clinical context. 


\section{INTRODUCTION}

During the course of cancer and the survivorship phase, many patients experience changes in their body image, a term which refers to the multifaceted psychological experience of embodiment $(1,2)$. Body image has been conceptualized as “a subjective picture of one's own physical appearance established both by self-observation and by noting the reactions of others." (3) and thus includes perceptions, emotions but also attitudes of a person towards their own body (4). According to the multidimensional conceptualization of body image, the disturbance of this construct (body image disturbance; BID) goes along with a negatively evaluated change in their appearance and psychological distress (5).

Body image represents an important issue among cancer patients as it is directly associated with both the disease and its treatment: For example, many cancer patients report chronic exhaustion (6) or undergo severe and sometimes irreversible body changes such as loss of a part of the body, disfigurement, skin changes, weight gain or loss, scars or hair loss (7). Such conditions in turn can adversely affect the body image by worsening overall vitality as well as the appraisal and feelings towards the body (8). Not surprisingly, studies across cancer populations could show that BID is significantly associated with depressive symptomatology (9-12). Furthermore, BID may play a mediating role between cancer-related factors including stigmatization and aspects of psychosocial wellbeing such as depression (13).

Previous studies have shown that many cancer survivors experience concerns about their appearance (14). Among all the late effects, body and physical alterations are frequently reported as a major concern (prevalence estimates vary from $30 \%$ to $60 \%$ ), especially in adolescent and young adult survivors $(15,16)$.

Despite the practical relevance of this concept, German language instruments to assess body image are not designed for oncological populations and thus may have limited applicability. For 
example, the German Body Image Questionnaire (Fragebogen zum Körperbild, FKB-20) (17)

contains several items asking about vitality which may be perceived as somewhat inappropriate by severely ill patients. Another questionnaire, the Body Appreciation Scale (BAS) (18), is more focused on positive body image and does not address the changes that patients with cancer tend to undergo.

A promising instrument that specifically addresses the situation of patients with cancer is the Body Image Scale (BIS) (7). This established and validated short self-report questionnaire assesses cancer-specific aspects of BID such as overall dissatisfaction with one's body, worries about physical and sexual attractiveness and feelings of being permanently disfigured by surgical treatment (4). While the questionnaire has been translated to several languages (19-24), no German version exists to date.

Using a sample of cancer survivors, we aimed to translate the BIS into German and validate the translated version using various approaches including confirmatory factors analyses (CFA) and item response theory (IRT). We thereby aimed to provide a German language tool to assess the specific aspects of BID in patients with cancer.

\section{METHODS}

\subsection{Participants}

We consecutively recruited cancer patients from two study centers (Leipzig and Berlin) between May 2018 and January 2020. In Leipzig, patients were recruited from the Psychosocial Counselling Center for cancer patients at the University Medical Center (25). In Berlin, patients were recruited from the wards of the Department for Hematology and Oncology and the Department of Radiation 
Oncology and Radiotherapy at Charité University Medical Center Berlin. Patients were informed about the study both orally and in writing.

We included all patients with a confirmed diagnosis of any malignancy according to ICD-10 (ICD-10: C00-97) who were at least 18 years old and proficient in German. Pregnant and breast feeding women were excluded.

\subsection{Ethics}

All participants provided written informed consent in accordance with the Declaration of Helsinki. The study was approved by the ethics committee at Leipzig University (reference number 090/18-ek). Data protection was secured in accordance with German data protection laws.

\subsection{Measures}

Sociodemographic and medical data were collected via patient self-report.

The Body Image Scale (BIS) is a validated self-report questionnaire assessing issues of selfperception, self-consciousness, loss of body wholeness, avoidance behavior and issues related to one's appearance within oncological contexts (7). Patients are asked to rate how they felt about 10 such aspects during the past week on a 4-point Likert Scale ranging from 0 ("not at all") to 3 ("very much"). Item 10 ("Have you been dissatisfied with the appearance of your scar?") also offers a "non-applicable" option for patients who do not have a scar. Item scores can be summed to a total score ranging from 0 to 30 , with higher scores indicating increased BID.

The validated German version of the BAS $(18,26)$ assesses positive body image in 13 items on a 5-point Likert scale ranging from 0 ("never") to 4 ("always"). The BAS shows excellent internal reliability (Cronbach's alpha $=0.94)$ and high correlations with measures of satisfaction with one's appearance (18). 
To assess patients' overall psychological symptom burden, we used (i) the NCCN Distress Thermometer (DT) assessing general distress during the past week on a 10-point visual analogue scale (27), (ii) the PHQ-9 to assess depressive symptomatology $(28,29)$ and (iii) the GAD-2 to assess anxious symptomatology $(30,31)$.

For the subgroup analyses, we used items from the problem checklist of the DT which indicated the occurrence of distressing problems (yes/no) with sexuality, appearance and fatigue (27). The item list was modified so that patients were asked to report problems that occurred within the previous month rather than week as this time frame was better suited to identify issues relevant for counselling sessions.

\subsection{Translation}

We used a state-of-the art translation procedure adapted from several guidelines to translate the BIS from English to German $(32,33)$. First, two translators independently created one German translation each. They then met to discuss differences in their translation and agreed upon one final German version.

Second, this German version was translated back into English by two other independent translators who did not know the original version. They also discussed differences in their translations to agree upon one final back translation. All translators were native speakers of the target language.

Third, the back translation was compared to the original questionnaire by an expert in psychosocial oncology research who is a native speaker of English. There was only one relevant difference between the back translation and the original version: Item 1 in the back translation used the word "insecure" rather than the original "self-conscious". The issue was discussed by the entire research team and consensus was reached that the German term used was the closest translation of "self-conscious" in this context. 


\section{5. $\quad$ Statistical procedures}

Statistical analyses were performed using IBM SPSS Statistics 24 (data validation and preparation for analysis, imputation of missing values), IBM SPSS Amos 24 (initial model evaluations), R statistics 4.0.2 using the packages lavaan (34) (confirmatory factor analysis), mirt (35) (IRT analyses) and userfriendlyscience (subgroup analyses) (36). All tests were two-tailed.

\subsubsection{Missing values}

Missing item values within each questionnaire (BIS, BAS, GAD-2 and PHQ-9) were replaced by the mean value of the answered items of the same questionnaire if more than $50 \%$ of the items were available. If more than $50 \%$ of the item values were missing from a scale, values were estimated on an item level using the Expectation Maximization algorithm (37). Imputed values outside the normal range were set to the nearest possible value. All other values were rounded to integers.

\subsubsection{Reliability and validity}

We conducted a confirmatory factor analysis (CFA) to test the one-factor solution of the original version. Model fit was evaluated with a combinational rule using the comparative fit index (CFI) and the standardized root mean square residual (SRMR). Models are considered acceptable if at least one of the two coefficients (CFI or SRMR) indicates acceptable fit (CFI $\geq 0.95$ or SRMR $\leq$ 0.06) (38). We computed additional fit-indices including the Tucker-Lewis-Index (TLI), the Root Mean Square Error of Approximation (RMSEA) and the Akaike's Information Criterion (AIC) in order to enable wide-ranging comparability to other studies.

In addition to model fit, we used CFA for additional psychometric properties including composite reliability ( $\mathrm{CR}$, adequate, if $\geq 0.6$ ), squared multiple correlations (SMC, also known as item reliabilities, adequate if $\mathrm{SMC}<\mathrm{CR}$ ) and the average variance extracted (AVE, adequate if $>$ 0.5) (39,40). For comparability, Cronbach's $\alpha$ was computed. 


\subsubsection{Item response theory}

IRT analyses were used to assess the discriminatory power and severity value of each individual item of the BIS. Severity parameters indicate the level of BID at which a particular item is likely to be endorsed: some items may be endorsed by patients with little or no BID, while other items are only affirmed by patients with very high levels of BID. Parameters concerning the discriminatory power indicate how specific an item is to the severity of BID at which it is most likely to be endorsed: Is it only affirmed by individuals with that exact level of BID or is similarly common to be affirmed by patients with somewhat higher or lower levels of BID? These results in turn can be used to identify (i) redundant items or answering options and (ii) items which poorly discriminate between high and low levels of BID.

To identify items that are redundant or show poor discriminatory power, we computed a 2parameter logistic (2PL) IRT model of dichotomized items. This model requires dichotomization of the items, which was achieved by merging the values of responses to 0 ("not at all" and "a little") and 1 ("quite a bit" and "very much"). The model then estimates the severity $b$ of BID (measured by the sum score of the questionnaire) at which the item has the highest discriminatory power, as well as the item's discriminatory power $a$ at this severity. Items with similar content and similar $b$ values could potentially be discarded to shorten the questionnaire. Any discrimination parameter $a$ $\geq 0.5$ was considered acceptable in this context (41). Results from the $2 \mathrm{PL}$ model were graphed as Item Characteristics Curves.

To detect potential floor effects of individual items, we also computed a 3PL model, which additionally includes a "guessing" parameter $c$, indicating the probability that the respective item is affirmed if all other items are negated. As the $a$ and $b$ parameters are not standardized in the 3PL model, we only report these parameters from the $2 \mathrm{PL}$ model for ease of interpretation and comparison to other studies. 
To assess whether the 4-point Likert-Structure of the questionnaire is appropriate, the discriminant power of individual answering options of each item was computed using graded response models and graphed using item trace lines. Item trace lines show the probability that a particular response is chosen with respect to the severity of the latent variable. Answering options that are not the most likely response at any severity can be discarded to simplify a questionnaire without losing accuracy. In contrast, if two answering options have a similar probability for the same area of severity, adding an additional option may improve the accuracy of the questionnaire.

\subsubsection{Convergent validity}

We assessed whether a priori hypothesized associations between the BIS score and other outcomes and patient characteristics could be verified.

To assess convergent validity with respect to other measures of body image, we computed the Pearson correlation of the BIS with the BAS. Since the two questionnaires measure related outcomes, we set the threshold for convergent validity to an absolute value of $r \geq 0.5$, which corresponds to a large effect size according to Cohen. As previous studies found BID to be positively associated with psychological symptom burden (12), we further estimated the correlation between the BIS total score and the PHQ-9, GAD-2 and DT.

Based on previous studies $(7,26)$, we further tested whether the BIS score was associated with various patient characteristics. For this purpose, we conducted t-tests for independent samples and used Hedges' g to assess effect size. In detail, we investigated differences in the BIS score according to age (below/above the median), gender or treatment (surgery, chemotherapy or radiotherapy) and the occurrence of distress due to fatigue, appearance or sexual problems (yes vs. no). 


\section{RESULTS}

\subsection{Participants}

Out of 877 eligible cancer survivors, 687 participated (overall response rate $78 \% ; 75 \%$ in Berlin and $78 \%$ in Leipzig) and sufficient data were available from $\mathrm{n}=677$. There were between $6.2 \%$ and 12.3 $\%$ missing values for each BIS item and between $8.6 \%$ and $11.7 \%$ for the BAS items, which were imputed as described in the methods section. Most patients had breast or prostate cancer and the mean time since diagnosis was 1.5 years (Table 1$)$.

Overall, the BIS questionnaire was filled out correctly by the majority of participants. However, the "non applicable" option for item 10 led to some misunderstandings, as several participants tried to tick "non-applicable" also for other items, even though no box with this option was provided for these items. 
Table 1. Sample characteristics ( $n=677)$.

\begin{tabular}{|c|c|}
\hline & $n(\%)^{2}$ \\
\hline Age, M (SD) [years] & $60.7(13.6)$ \\
\hline$<40$ years & $52(8)$ \\
\hline 40 to $<50$ years & $89(13)$ \\
\hline 50 to $<60$ years & $160(24)$ \\
\hline 60 to $<70$ years & $178(26)$ \\
\hline 70 to $<80$ years & $159(23)$ \\
\hline$\geq 80$ years & $39(6)$ \\
\hline \multicolumn{2}{|l|}{ Gender } \\
\hline female & $390(58)$ \\
\hline male & $287(42)$ \\
\hline \multicolumn{2}{|l|}{ Marital status } \\
\hline single & $101(15)$ \\
\hline married & $408(61)$ \\
\hline divorced / separated & $107(16)$ \\
\hline widow/er & $56(8)$ \\
\hline Children & $559(83)$ \\
\hline \multicolumn{2}{|l|}{ Education } \\
\hline$\leq 10$ years of education & $401(60)$ \\
\hline$>10$ years of education & $263(40)$ \\
\hline \multicolumn{2}{|l|}{ Employment status } \\
\hline currently working & $258(40)$ \\
\hline unemployed & $44(7)$ \\
\hline retired & $319(49)$ \\
\hline other situation & $32(5)$ \\
\hline \multicolumn{2}{|l|}{ Cancer site } \\
\hline breast & $226(33)$ \\
\hline Prostate & $115(17)$ \\
\hline head and neck & $52(8)$ \\
\hline skin & $45(7)$ \\
\hline female genital organs & $39(6)$ \\
\hline central nervous system & $35(5)$ \\
\hline hematological & $34(5)$ \\
\hline lung & $31(5)$ \\
\hline colorectal & $25(4)$ \\
\hline kidney/urinary tract & $18(3)$ \\
\hline gastroesophageal & $16(2)$ \\
\hline other & $41(6)$ \\
\hline Metastases & $118(18)$ \\
\hline Time since diagnosis M (SD) [months] & $18.88(34.11)$ \\
\hline \multicolumn{2}{|l|}{ Treatment } \\
\hline surgery & $508(77)$ \\
\hline chemotherapy & $258(39)$ \\
\hline radiotherapy & $468(71)$ \\
\hline hormone therapy & $105(16)$ \\
\hline other & $56(9)$ \\
\hline
\end{tabular}

${ }^{a}$ unless otherwise specified. Abbreviations: M, mean;

$\mathrm{SD}$, standard deviation 


\subsection{Reliability and validity}

The test reliability was Cronbach's $\alpha=0.92$ (95\%CI 0.91, 0.93). The composite reliability was 0.92 and the construct explained on average more than a half of variance that was due to measurement (Figure 1). SRMR was 0.047 and the criteria for acceptable model fit were therefore fulfilled. In order to enable the comparability of our results with other studies, we additionally report the following coefficients: TLI $=0.88$, RMSEA=0.12, and AIC=12746.9. 


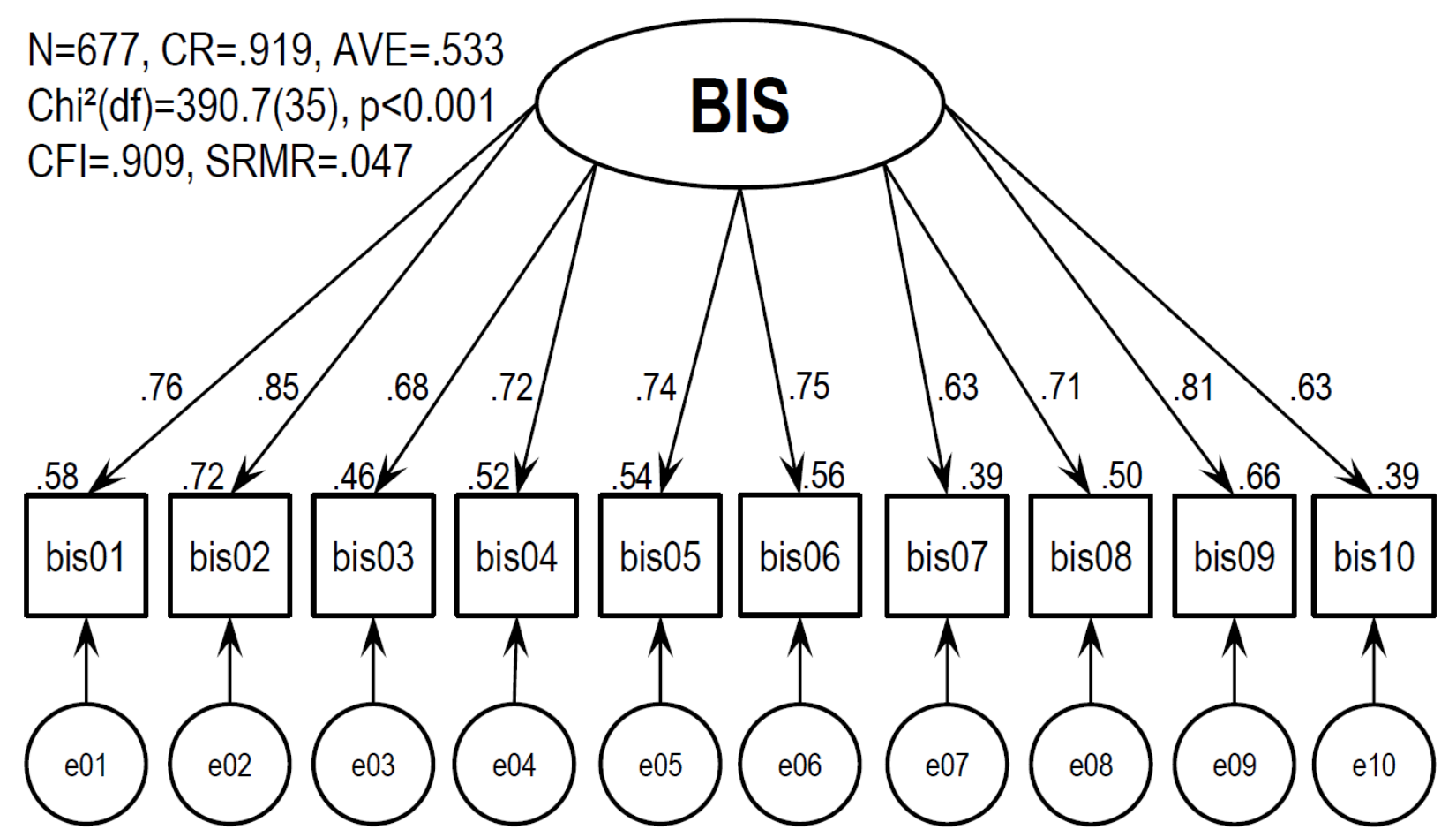

Figure 1. Confirmatory factor analysis (CFA) of the Body Image Scale (BIS). Rectangles represent manifest variables (items); ovals represent latent variables (factors, composites, constructs); values above the rectangles show squared multiple correlations (SMC, item reliabilities); values at straight arrows show standardized regression weights (standardized loadings). Abbreviations: N sample size; AVE average variance extracted; CR composite reliability; $\mathrm{Chi}^{2}$ chi squared statistic (minimum discrepancy function); df degrees of freedom; $\mathrm{p}$ type-I-error-probability; CFI comparative fit index; SRMR standardized root mean square residual; model fit is considered acceptable if $\mathrm{CFI} \geq 0.95$ or if $\mathrm{SRMR} \leq 0.06$. 


\subsection{Item response theory analyses}

IRT analyses showed that all items had a discriminating power above the established threshold of $b=0.5$ (41). Item 9 (dissatisfaction with one's body) showed the highest discrimination power ( $a$ $=4.02$ ) and item 3 (dissatisfaction with one's appearance when dressed) showed the lowest ( $a=$ 1.95; Table 2).

Item 7 had an optimal discriminatory ability at the highest severity level $(b=2.06)$ while item 6 optimally discriminated at the lowest severity $(b=0.89)$ (Figure 2), i.e., dichotomized items discriminate best between very high BID and medium BID.

The graded response model showed category thresholds ranging from $b 1=0.06$ to $b 3=2.51$ (see table, Supplemental Digital Content 1, which shows Slope (a) and category thresholds (b1-b3) for a graded response model of the Body Image Scale). Visual inspection of the item trace lines for all items showed that all four answering options had an area of severity where the respective option was the most likely option to be chosen (see Figure, Supplemental Digital Content 2, which shows item trace lines for all items of the BIS).

The test information curve showed that the questionnaire measured BID across a range of standardized severity values $\theta$ from approximately -1.3 to 3.7 with a maximum at $\theta=1.36$ (see figure, Supplemental Digital Content 3, which shows the test information curve of the BIS). 
Table 2. Item statistics for the Body Image Scale including parameters from the 2PL IRT model $(\mathrm{n}=677)$.

\begin{tabular}{llccccc}
\hline Item & Description & Mean (SD) & Skewness & Kurtosis & $\mathrm{b}$ & $\mathrm{a}$ \\
\hline 1 & Self-conscious about appearance & $0.63(0.81)$ & 1.21 & 0.80 & 1.30 & 2.66 \\
2 & Subjective physical attractiveness & $0.69(0.86)$ & 1.06 & 0.22 & 1.04 & 3.37 \\
3 & Appearance when dressed & $0.47(0.76)$ & 1.55 & 1.57 & 1.58 & 1.95 \\
4 & Loss of femininity / masculinity & $0.47(0.82)$ & 1.76 & 2.18 & 1.32 & 3.31 \\
5 & Difficulty looking at oneself naked & $0.52(0.85)$ & 1.57 & 1.51 & 1.28 & 2.86 \\
6 & Loss of sexual attractiveness & $0.79(0.96)$ & 1.00 & -0.10 & 0.89 & 3.39 \\
7 & Avoidance behavior & $0.21(0.53)$ & 2.79 & 7.99 & 2.06 & 2.73 \\
8 & Feeling less whole & $0.79(0.90)$ & 0.86 & -0.25 & 0.97 & 2.36 \\
9 & Dissatisfaction with one's body & $0.72(0.84)$ & 0.96 & 0.10 & 1.01 & 4.02 \\
10 & Appearance of one's scar & $0.54(0.79)$ & 1.44 & 1.45 & 1.52 & 2.19 \\
\hline
\end{tabular}

Abbreviations: SD, standard deviation; b Severity, a Discrimination 


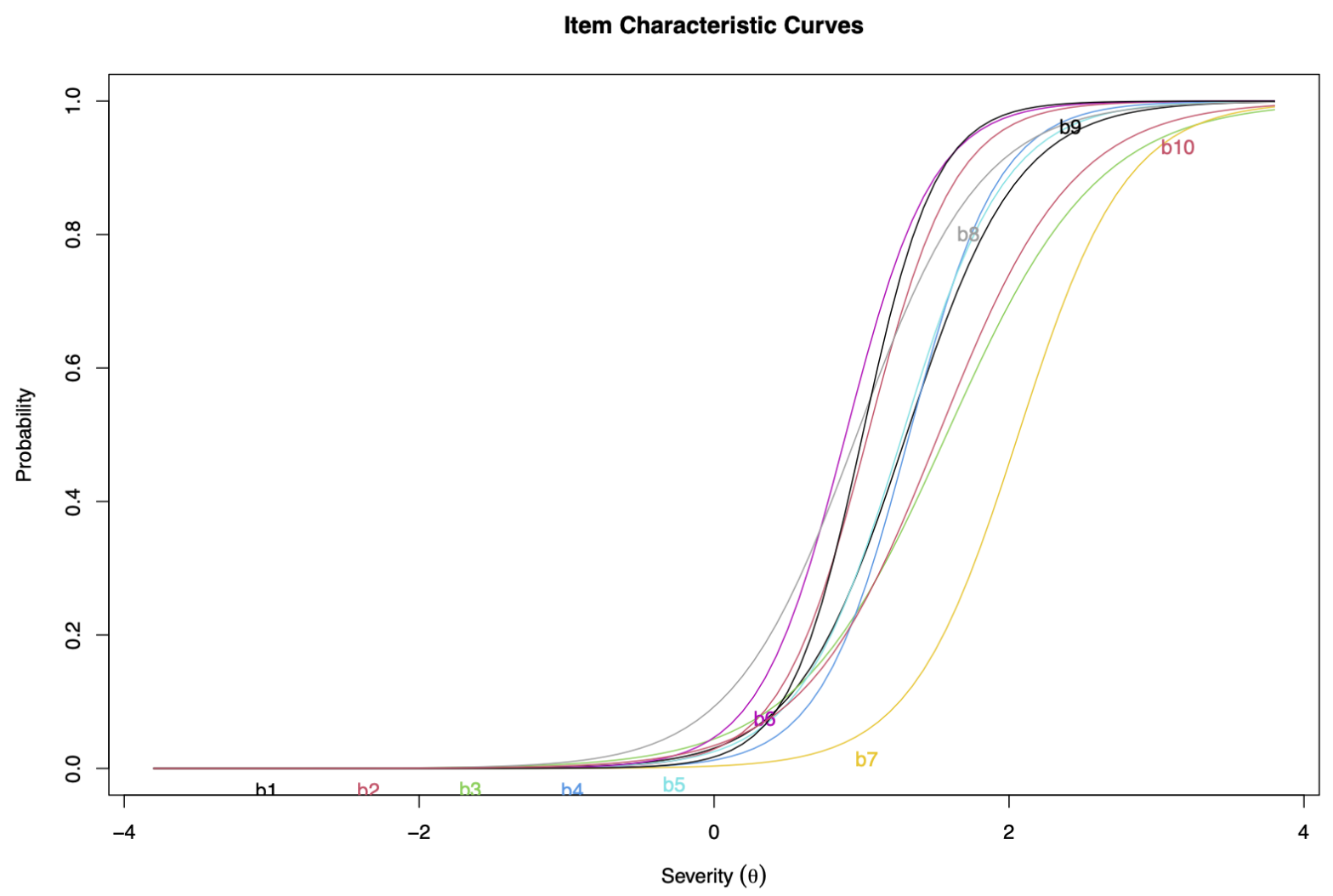

Figure 2. Item Characteristic Curves from a two-parameter logistic (2PL) model of dichotomized Body Image Scale Items. The slope of the curve represents the discriminatory ability of the item $(a)$. The position along the $\mathrm{x}$-axis $(b)$ represents the severity of BID at which the item has the highest discriminatory ability, i.e. the steepest slope. 
According to the 3PL model, only item 6 (sexual attractiveness) showed a relevant probability (approx. 10\%) to be affirmed, even if all other items took the lowest possible value [data not shown].

\subsection{Convergent validity}

The of the BIS demonstrated good convergent validity. In detail, the BIS was strongly correlated with the BAS, with $r=-0.618$ (95\%CI: $-0.663,-0.570 ; p<0.001)$. It also was also significantly correlated with psychological symptom burden, i.e., depressive symptoms $(r=0.490 ; 95 \% \mathrm{CI}$ : $0.431,0.546 ; \mathrm{p}<0.001)$, anxious symptoms ( $\mathrm{r}=0.476 ; 95 \% \mathrm{CI}: 0.416,0.532 ; \mathrm{p}<0.001)$ and general distress $(\mathrm{r}=0.315 ; 95 \%-\mathrm{CI}: 0.243,0.382, \mathrm{p}<0.001)$.

We found that patients who were younger, female, had undergone chemotherapy and those who were distressed by fatigue, their appearance or sexual problems reported higher BIS values (Table 3). Effect sizes ranged from small (gender) to large (distressed by appearance). There was no significant difference in BIS scores with respect to surgery or radiotherapy. 
Table 3. Body Image Scale sum scores for different subgroups ( $\mathrm{n}=677)$.

\begin{tabular}{llcrccc}
\hline Variable & Group & $\mathrm{n}$ & Mean & SD & $\mathrm{p}$ & ES $^{\mathrm{a}}$ \\
\hline Age & above median $^{\mathrm{b}}$ & 321 & 4.50 & 5.43 & $<0.001$ & 0.41 \\
& below median $^{\mathrm{b}}$ & 356 & 7.02 & 6.61 & & \\
Gender & women & 390 & 6.54 & 6.45 & $<0.001$ & 0.27 \\
& men & 287 & 4.86 & 5.71 & & \\
Distressed by fatigue & yes & 525 & 6.46 & 6.36 & $<0.001$ & 0.49 \\
& no & 141 & 3.48 & 4.91 & & \\
Distressed by appearance & yes & 153 & 10.29 & 7.32 & $<0.001$ & 1.07 \\
& no & 466 & 4.28 & 4.96 & & \\
Distressed by sexual problems & yes & 182 & 8.38 & 7.23 & $<0.001$ & 0.62 \\
& no & 440 & 4.66 & 5.35 & & \\
Surgery & yes & 508 & 6.06 & 6.20 & 0.095 & 0.16 \\
& no & 152 & 5.09 & 6.26 & & \\
Chemotherapy & yes & 258 & 7.61 & 6.79 & $<0.001$ & 0.48 \\
& no & 402 & 4.70 & 5.54 & & \\
\hline Radiotherapy & yes & 468 & 5.55 & 6.12 & 0.080 & 0.15 \\
& no & 194 & 6.49 & 6.41 & & \\
\hline
\end{tabular}

a absolute value of Hedges' g: 0.2 (small effect). 0.5 (medium effect). 0.8 (large effect)

$\mathrm{b}$ median age $=62$ years

Abbreviations: SD, standard deviation; ES, effect size 


\section{DISCUSSION}

This study aimed to translate the BIS into German and to test its psychometric properties. Our analyses confirmed that the German version is a valid tool for assessing BID in cancer survivors. This BIS translation showed excellent composite reliability and good convergent validity. All items showed good item response characteristics. The one-factor solution of the original questionnaire could be confirmed.

\subsection{Evaluation of the psychometric findings}

The original English language questionnaire showed good reliability (Cronbach's alpha ranging from 0.78 to 0.85 ) and test-retest reliability after one month (7). In our German translation, reliability was similar to the original BIS and its other translations $(7,19,20)$. In addition to the psychometric evaluation performed in previous translation studies, we used a state-of-the-art translation method and performed IRT analyses, which enabled us to check for redundant items, informational value of each item and the questionnaire as a whole and the applicability of the (Likert-)structure of the scale.

The model showed good construct reliability with excellent composite reliability and acceptable model fit for a one-factor solution. This is consistent with the original version and translations to other languages $(7,19,20)$.

Our IRT analyses revealed that all items showed very high discriminatory power, well above the recommended threshold (41). However, most items discriminated best between very high BID and high BID. This suggests that the BIS may not be sensitive enough to identify individuals with low BID.

Our IRT analyses also identified items that could be omitted to shorten the questionnaire. While items 6 and 7 showed optimal discrimination at the lowest and highest severity, respectively, the 
other items fall into three severity clusters: items 2,8 and $9(b=0.97-1.04)$, items 1,4 and $5(b=$ 1.28-1.32), and items 3 and $10(b=1.52-1.58)$. Selecting only the item with the best discriminatory ability from each cluster, it would be feasible to shorten the questionnaire to 5 items $(4,6,7,9,10)$, e.g. when questionnaire space is very limited. However, considering that items within each cluster ask about qualitatively different aspects of BID, we currently do not recommend shortening the questionnaire for most purposes.

Visual inspection of the graded response IRT models showed that the 4-point Likert-structure is appropriate and we currently do not recommend changing it to a 3-point or 5-point scale. As the additional column for item 10 option "non-applicable" appeared to be misleading, we recommend providing the non-applicable option in a separate line rather than a separate column, as was the layout of the original English version. For additional clarity, it is also possible to insert a question after item 9, asking about whether or not the cancer treatment left a scar and only offer item 10 if the question is affirmed. A German version of the questionnaire with the suggested modifications can be found in the supplemental materials (see document, Supplemental Digital Content 4, which contains the German versions of the BIS with and without the optional inserted questions).

As hypothesized, the BIS score was strongly correlated with another body image instrument (BAS), indicating good convergent validity. In this context, the group effects showed significantly worse body image of those who are younger, female and distressed by either physical condition, outer appearance or sexual problems. These results are largely in line with previous studies $(7,26,42)$, lending further evidence to the convergent validity of the translated instrument.

Other than previous studies, we were not able to find a significant difference in BIS score between patients with/without surgery or radiotherapy, respectively. One of the reasons for this finding may be that the group of patients with surgery was heterogeneous and ranged from procedures that leave only minor external marks such as endoscopic procedures or breast 
conserving surgery to patients with major alterations to their bodies such as mastectomy, amputations, tracheotomy or stomas.

As expected from previous studies (12), BIS sum scores significantly correlated with depression, anxiety and general distress, all of which were medium-sized effects. Again, this demonstrates good convergent validity. Furthermore, this underlines the importance of BID as an issue that is often accompanied by clinically relevant levels of psychosocial distress.

\subsection{Clinical implications}

The German version of the BIS can be used to evaluate BID in clinical settings, e.g. as part of psychosocial counselling or psychotherapeutic interventions, but also be useful for nurses and other therapists. Body-related issues are often shameful or difficult to put into words. The BIS may therefore facilitate a conversation about topics which patients may not mention on their own. By providing pre-formulated statements, body-related issues may be more accessible to patients and the BIS may serve as a tool for patients to contemplate these aspects of wellbeing before a given consultation.

Some patients are less inclined towards verbal psychotherapy and alternative forms of therapy may be more suitable. By shifting the focus to body-related issues, the BIS may serve as a gateway to non-verbal elements of therapy such as breathing techniques, physical exercise, relaxation techniques such as autogenic training or progressive muscle relaxation, which have been shown to be effective for treating BID (43). On the other hand, it may be important to assess BID before using such techniques as patients with high levels of BID or even traumatic physical experiences may suffer severe distress when undergoing body-focused therapy. We therefore recommend that BID in cancer survivors should only be treated by trained psycho-oncologists.

Cancer and its treatment not only affect the patient's sense of physical or sexual attractiveness but also other aspects of body image. During a variety of treatments (from intravenous therapies to 
intensive care and surgery), patients may make voluntary or involuntary experiences of touch, of manipulating or breaking their skin and their physical boundaries. The BIS captures parts of these aspects through questions such as item 8 ("Have you been feeling the treatment has left your body less whole?"). The BIS thus addresses aspects of BID beyond physical attractiveness.

\subsection{Implications for future research}

The German version of the BIS allows for assessment of BID in research contexts, e.g. as a clinical end-point or as a mediator variable in studies on the psychosocial wellbeing of cancer survivors (44). It can also facilitate the development and implementation of clinical interventions aimed at reducing BID. This may be particularly relevant as BID has been linked to lower quality of life and higher risk of depression (12).

Normative data from representative oncological samples are needed to put individual test results into context. The association with overall psychosocial symptom burden and distress should be explored further to recommend categorization of sum scores into different severity levels. Such studies could also investigate if there are specific aspects of BID that show particularly strong links to other aspects of psychosocial distress. As previous studies suggest that tools such as the BIS may be particularly useful in adolescent and young adult cancer survivors, future studies should seek to recruit representative (sub-)samples from this population (42).

The BIS could also be used as a screening tool in high-risk groups, which would require studies identifying an optimal cutoff value. Further research would then be needed to assess whether the BIS is sensitive enough to detect low levels of BID in mass screening. In addition, an ultra-short screener consisting of 2-3 items could be developed. Our results provide a useful starting point for selecting items to be used in such a screener. In addition, longitudinal studies could assess the questionnaire's sensitivity to change to confirm its usefulness in intervention studies. 
As a questionnaire designed for people with physical impairments, the BIS can also be used in patients with other chronic diseases and has been successfully applied for conditions such as inflammatory bowel disease (45).

\subsection{Strengths and Limitations}

Strengths of our study include the state-of-the-art translation process, the large sample size and the sophisticated analyses including confirmatory factor analyses and item response analyses. The latter enabled us to demonstrate the high discriminatory power of individual items, confirm the utility of the 4-point Likert-structure and offer an approach to shortening the questionnaire if needed.

Our results ought to be interpreted in light of the following limitations: The study used a convenience sample, mostly recruited from a psychosocial counselling center. Although it is a large sample, including patients across the entire oncological spectrum, it should not be mistaken for a representative epidemiological sample. It is likely that our sample showed higher levels of BID than the average oncological population, as many of the patients actively sought treatment for psychosocial issues. Although these data were appropriate to validate the questionnaire, our mean values cannot serve as normative data. As our study design was cross-sectional, we were not able to assess test-retest reliability or sensitivity to changes over time. Accordingly, associations with other scales cannot be interpreted in a causal manner.

\subsection{Conclusion}

The German version of the BIS is a useful and valid tool to assess BID in patients with cancer both in research and in clinical practice. Future research may provide reference values or determine clinically relevant cut-offs to ease and improve interpretation of the scores within clinical care. 


\section{REFERENCES}

1. Cash TF. Body image: Past, present, and future. Body Image 2004;1:1-5.

2. Hopwood P, Hopwood N. New challenges in psycho-oncology: An embodied approach to body image. Psychooncology 2019;28:211-18.

3. Merriam-Webster. Body image. Merriam-Webster Medical Dictionary. 2020 [cited 2020 May 10]. Available from: https://www.merriam-webster.com/medical/body image

4. Muzzatti B, Annunziata MA. Body image assessment in oncology: an update review. Support Care Cancer 2017;25:1019-29.

5. Rhoten BA. Body image disturbance in adults treated for cancer - A concept analysis. J Adv Nurs 2016;72:1001-11.

6. Wang XS, Zhao F, Fisch MJ, O’Mara AM, Cella D, Mendoza TR, Cleeland CS. Prevalence and characteristics of moderate to severe fatigue: A multicenter study in cancer patients and survivors. Cancer 2014;120:425-32.

7. Hopwood P, Fletcher I, Lee A, Al Ghazal S. A body image scale for use with cancer patients. Eur J Cancer 2001;37:189-97.

8. Albani C, Blaser G, Geyer M, Schmutzer G, Hinz A, Bailer H, Grulke N, Brähler E.

[Validation and standardization of the "Questionnaire for Assessing Subjective Physical Well-Being" by Kolip and Schmidt in a representative German sample]. Psychother Psychosom Med Psychol 2006;56:172-81.

9. Sharpe L, Patel D, Clarke S. The relationship between body image disturbance and distress in colorectal cancer patients with and without stomas. J Psychosom Res 2011;70:395-402.

10. Bullen TL, Sharpe L, Lawsin C, Patel DC, Clarke S, Bokey L. Body image as a predictor of 25 
psychopathology in surgical patients with colorectal disease. J Psychosom Res 2012;73:45963.

11. Przezdziecki A, Sherman KA, Baillie A, Taylor A, Foley E, Stalgis-Bilinski K. My changed body: Breast cancer, body image, distress and self-compassion. Psychooncology 2013;22:1872-79.

12. Begovic-Juhant A, Chmielewski A, Iwuagwu S, Chapman LA. Impact of Body Image on Depression and Quality of Life Among Women with Breast Cancer. J Psychosoc Oncol 2012;30:446-60.

13. Esser P, Mehnert A, Johansen C, Hornemann B, Dietz A, Ernst J. Body image mediates the effect of cancer-related stigmatization on depression: A new target for intervention. Psychooncology 2018;27:193-98.

14. White CA. Body image and cancer: a heuristic cognitive behavioural model. Psychooncology 2000;9:183-92.

15. Fan S-Y, Eiser C. Body image of children and adolescents with cancer: A systematic review. Body Image 2009;6:247-56.

16. Lehmann V, Hagedoorn M, Tuinman MA. Body image in cancer survivors: a systematic review of case-control studies. J Cancer Surviv 2015;9:339-48.

17. Clement U, Löwe B. Die Validierung des FKB-20 als Instrument zur Erfassung von Körperbildstörungen bei psychosomatischen Patienten. [The validation of the Body-Image Questionnaire (BIQ): A German-language instrument for assessing body image disturbances in patients with psy. Psychother Psychosom Med Psychol 1996;46:254-59.

18. Avalos L, Tylka TL, Wood-Barcalow N. The Body Appreciation Scale: Development and psychometric evaluation. Body Image 2005;2:285-97. 
19. Gómez-Campelo P, Bragado-Álvarez C, Hernández-Lloreda MJ, Sánchez-Bernardos ML. The Spanish version of the Body Image Scale (S-BIS): psychometric properties in a sample of breast and gynaecological cancer patients. Support Care Cancer 2014;23:473-81.

20. van Verschuer VMT, Vrijland WW, Mares-Engelberts I, Klem TMAL. Reliability and validity of the Dutch-translated Body Image Scale. Qual Life Res 2015;24:1629-33.

21. Brédart A, Swaine Verdier A, Dolbeault S. Traduction/adaptation française de l'échelle "Body Image Scale" (BIS) évaluant la perception de l'image du corps chez des femmes atteintes de cancer du sein. Psycho-Oncologie 2007;1:24-30.

22. Cheli S, Agostini A, Herd-Smith A, Caligiani L, Martella F, Fioretto L. The Italian version of Body Image Scale Reliability and Sensitivity in a sample of breast cancer patients. Psicoter Cogn e Comport 2016;22:65-79.

23. Moreira H, Silva S, Marques A, Canavarro MC. The Portuguese version of the Body Image Scale (BIS) - psychometric properties in a sample of breast cancer patients. Eur J Oncol Nurs 2010;14:111-18.

24. Karayurt Ö, Edeer AD, Süler G, Dorum H, Harputlu D, Vural F, Ceylanim Ü. Psychometric Properties of the Body Image Scale in Turkish Ostomy Patients. Int J Nurs Knowl 2015;26:127-34.

25. Lehmann-Laue A, Danker H, Schröter K, Friedrich M, Mehnert A, Ernst J. Psychosoziale Versorgung von Krebspatienten in einer Krebsberatungsstelle an einem Universitätsklinikum. Psychother Psychosom Med Psychol 2019;69:20-28.

26. Swami V, Stieger S, Haubner T, Voracek M. German translation and psychometric evaluation of the Body Appreciation Scale. Body Image 2008;5:122-27.

27. Mehnert A, Müller D, Lehmann C, Koch U. Die deutsche Version des NCCN Distress- 
Thermometers. Zeitschrift für Psychiatr Psychol und Psychother 2006;54:213-23.

28. Kroenke K, Spitzer RL, Williams JBW. The PHQ-9: Validity of a brief depression severity measure. J Gen Intern Med 2001;16:606-13.

29. Löwe B, Gräfe K, Zipfel S, Witte S, Loerch B, Herzog W. Diagnosing ICD-10 depressive episodes: Superior criterion validity of the Patient Health Questionnaire. Psychother Psychosom 2004;73:386-91.

30. Kroenke K, Spitzer RL, Williams JBW, Monahan PO, Löwe B. Anxiety Disorders in Primary Care: Prevalence, Impairment, Comorbidity, and Detection. Ann Intern Med 2007;146:317.

31. Löwe B, Wahl I, Rose M, Spitzer C, Glaesmer H, Wingenfeld K, Schneider A, Brähler E. A 4-item measure of depression and anxiety: Validation and standardization of the Patient Health Questionnaire-4 (PHQ-4) in the general population. J Affect Disord 2010;122:86-95.

32. Behling O, Law K. Translating Questionnaires and Other Research Instruments: Problems and Solutions. Thousand Oaks, CA: Sage Publications; 2000.

33. World Health Organization. Process of translation and adaptation of instruments. 2021 [cited 2021 Apr 17]. Available from:

https://www.who.int/substance_abuse/research_tools/translation/en/

34. Rosseel Y. Lavaan: An R package for structural equation modeling and more. Version 0.5-12 (BETA). J Stat Softw 2012;48:1-36.

35. Chalmers RP. mirt : A Multidimensional Item Response Theory Package for the R Environment. J Stat Softw [Internet] 2012;48. Available from:

http://www.jstatsoft.org/v48/i06/ 
36. Peters G. userfriendlyscience: Quantitative analysis made accessible. 2015 [cited 2021 Feb 19]. Available from: https://userfriendlyscience.com

37. Dempster AP, Laird NM, Rubin DB. Maximum likelihood from incomplete data via the EM algorithm. J R Stat Soc Ser B 1977;39:1-22.

38. Hu L, Bentler PM. Cutoff criteria for fit indexes in covariance structure analysis:

Conventional criteria versus new alternatives. Struct Equ Model A Multidiscip J 1999;6:155.

39. Fornell C, Larcker DF. Structural Equation Models with Unobservable Variables and Measurement Error: Algebra and Statistics. J Mark Res 1981;18:382-88.

40. Bagozzi RP, Yi Y. On the evaluation of structural equation models. J Acad Mark Sci 1988;16:74-94.

41. Baker FB. The Basics of Item Response Theory. Washington, DC: ERIC Clearinghouse on Assessement and Evaluation; 2001. 172 p.

42. Zucchetti G, Bellini S, Bertolotti M, Bona F, Biasin E, Bertorello N, Tirtei E, Fagioli F. Body Image Discomfort of Adolescent and Young Adult Hematologic Cancer Survivors. J Adolesc Young Adult Oncol 2017;6:377-80.

43. Mehnert A, Veers S, Howaldt D, Braumann KM, Koch U, Schulz KH. Effects of a physical exercise rehabilitation group program on anxiety, depression, body image, and health-related quality of life among breast cancer patients. Onkologie 2011;34:248-53.

44. Esser P, Mehnert A, Johansen C, Hornemann B, Dietz A, Ernst J. Body image mediates the effect of cancer-related stigmatization on depression: A new target for intervention. Psychooncology 2018;27:193-98. 
45. McDermott E, Moloney J, Rafter N, Keegan D, Byrne K, Doherty GA, Cullen G, Malone K, Mulcahy HE. The body image scale: A simple and valid tool for assessing body image dissatisfaction in inflammatory bowel disease. Inflamm Bowel Dis 2014;20:286-90. 